\title{
Epidemiología del tabaquismo en Europa
}

Esteve Fernández, MD, PhD, (1) Anna Schiaffino, BSc, ${ }^{(1)}$ Josep M Borrás, MD, PhD.(1)

\begin{abstract}
Fernández E, Schiaffino A, Borrás JM.
Epidemiología del tabaquismo en Europa. Salud Publica Mex 2002;44 supl 1:S11-S19.

El texto completo en inglés de este artículo está disponible en: http://www.insp. mx/salud/index.html
\end{abstract}

\section{Resumen}

El tabaco es la causa de mortalidad prevenible más importante en los países europeos, en los que da cuenta de más de medio millón de muertes anuales. El objetivo de este trabajo es revisar la epidemiología del tabaquismo en Europa, atendiendo a la visión de conjunto sobre el impacto del tabaquismo, a la prevalencia del consumo y a su evolución en la última década, así como revisar otras características relacionadas con la difusión y mantenimiento del tabaquismo: la producción y los precios del tabaco. Treinta de cada 100 europeos adultos fuma cigarrillos a diario. En los países de la Unión Europea se estima que 1 de cada 10 adultos fumado res mo rirá a causa de los efectos del tabaco, razón que se eleva a 1 de cada 5 en los países del este de Europa. La prevalencia de fumadores desciende entre los hombres adultos en algunos países de la Unión Europea, mientras que aumenta en las mujeres, sobre to do jóvenes, del sur y del este de Europa. Se debe insistir en las medidas para controlar el consumo de tabaco y prevenirlo, tales como la prohibición del consumo en lugares públicos, la prohibición real de la publicidad directa e indirecta, la reducción del cultivo, o el incremento de precios. Estas acciones se deben diseñar, coordinar y desarrollar en y desde los diferentes sectores involucrados en la lucha contra el tabaco, con la participación de redes civiles impulsadas desde los diferentes niveles administrativos (local, regional, nacional) con la decisiva participación de organismos y organizaciones supranacionales. El texto completo en inglés de este artículo está disponible en: http://www.insp.mx/salud/ index.html

Palabras clave: tabaco; epidemia; tendencias; prevalencia; mortalidad; Europa

\author{
Fernández E, Schiaffino A, Borrás JM. \\ Epidemiology of smoking in Europe. \\ Salud Publica Mex 2002;44 suppl 1:S11-S19. \\ The English version of this paper \\ is available at: http://www.insp.mx/salud/index.html
}

\begin{abstract}
A bstract
Tobacco is the most important preventable cause of mortality in European countries, accounting for over half a million deaths per year. A review is presented on the epidemiology of to bacco smoking in Europe, using a comprehensive approach on the health effects of smoking, the prevalence of tobacco consumption, and its evolution in the past decade.Tobacco industry efforts to promote and maintain smoking through production and princing are also reviewed. Thirty out of every 100 European adults smoke everyday and one of every ten adults smokers will die from tobacco smoking; a higher ratio of 1:15 is found in Eastern Europe. The prevalence of smokers is decreasing among young adults in so me European countries, while it is increasing among young women in Southern and Eastern Europe. Smoking cessation and prevention interventions should be implemented, such as banning smoking in public areas, banning direct and indirect advertising of to bacco products, crop reduction, and rising cigarette prices. These interventions should be designed, coordinated, and developed by and among the different sectors involved in tobacco control initiatives, together with social network at the local, regional, and national levels, with the support of national and international organizations. The English version of this paper is available at: http://www.insp.mx/salud/index.html
\end{abstract}

Key words: to bacco; epidemic; trends; prevalence; mortality; Europe

(1) Servicio de Prevención y Control del Cáncer. Instituto Catalán de 0 ncología, Barcelona, España.

Fecha de recibido: 30 de marzo de 2001 - Fecha de aprobado: 11 de junio de 2001

Solicitud de sobretiros: Dr. Esteve Fernández. Instituto Catalán de 0 ncología. Servicio de Prevención y Control del Cáncer. Avenida Gran Vía s/n Km 2,7 08907 L’H ospitalet (Barcelona), España. Correo electrónico: efernandez@ ico.scs.es 
c on el motivo del Día Mundial sin Tabaco del año 2000, se reunieron en Lisboa, Portugal, delegados de 18 países europeos con el objetivo de identificar los retos para el nuevo milenio en la prevención y control del tabaquismo en Europa. Las conclusiones, plasmadas en la "Declaración de Lisboa", ${ }^{1}$ hacían referencia, entre otros importantes temas, a la regulación de la producción de tabaco, a la integración del control del tabaco en las nuevas políticas de salud pública europeas, a la protección de los no fumadores o a la colaboración multidisciplinar para la promoción del abandono del hábito tabáquico y para su prevención entre niños y adolescentes en el ámbito europeo.

Es bien conocido que el tabaco es la causa de mortalidad prevenible más importante en los países europeos, en los que da cuenta de más de medio millón de muertes anuales, ${ }^{2}$ de las cuales la mitad corresponde a personas de menos de 70 años. ${ }^{3}$ A pesar de que el problema del tabaquismo es común y de que se están realizando esfuerzos comunes en la región europea, la epidemia del tabaquismo presenta características particulares en los diferentes países europeos, y que se deben tener en cuenta en los estudios sobre la dinámica del tabaquismo. ${ }^{4-6}$

El objetivo de este trabajo es revisar la epidemiología del tabaquismo en Europa, atendiendo a la visión de conjunto sobre el impacto del tabaquismo, a la prevalencia del consumo y a su evolución en la última década, así como revisar otras características relacionadas con la difusión y mantenimiento del tabaquismo: la producción y los precios del tabaco.

\section{Material y métodos}

La información recogida se refiere, siempre que ha sido posible, a los siguientes 22 países europeos: a) Alemania, Austria, Bélgica, Dinamarca, España, Finlandia, Francia, Grecia, Irlanda, Italia, Luxemburgo, Países Bajos, Portugal, Reino Unido y Suecia (países pertenecientes a la Unión Europea); b) Islandia, Noruega y Suiza (como otros países norte y centro europeos); y c) Bulgaria, Hungría, Polonia y Rumania (países del este de Europa).

La información se ha recopilado y elaborado a partir de diferentes fuentes y bases de datos públicos: la base de datos de mortalidad de la Organización Mundial de la Salud (OMS) ${ }^{7}$ y su Informe "Tabaco o Salud",, 8 y otras fuentes que se citan oportunamente en el texto.

\section{Resultados y discusión}

Impacto del tabaquismo: mortalidad por enfermedad isquémica del corazón y cáncer de pulmón

La mortalidad por enfermedad isquémica cardiaca y por cáncer de pulmón puede utilizarse como una buena aproximación al impacto del tabaquismo en los países europeos. En las figuras 1 y 2 se muestran las tasas estandarizadas (población mundial) de mortalidad para estas enfermedades en los 22 países considerados durante el año 1996 (en algunos países la tasa corresponde a 1995 o a 1994). Podemos observar claramente un patrón norte-sur en la mortalidad por enfermedad isquémica del corazón (figura 1), tanto en hombres como en mujeres, con tasas cinco o seis veces

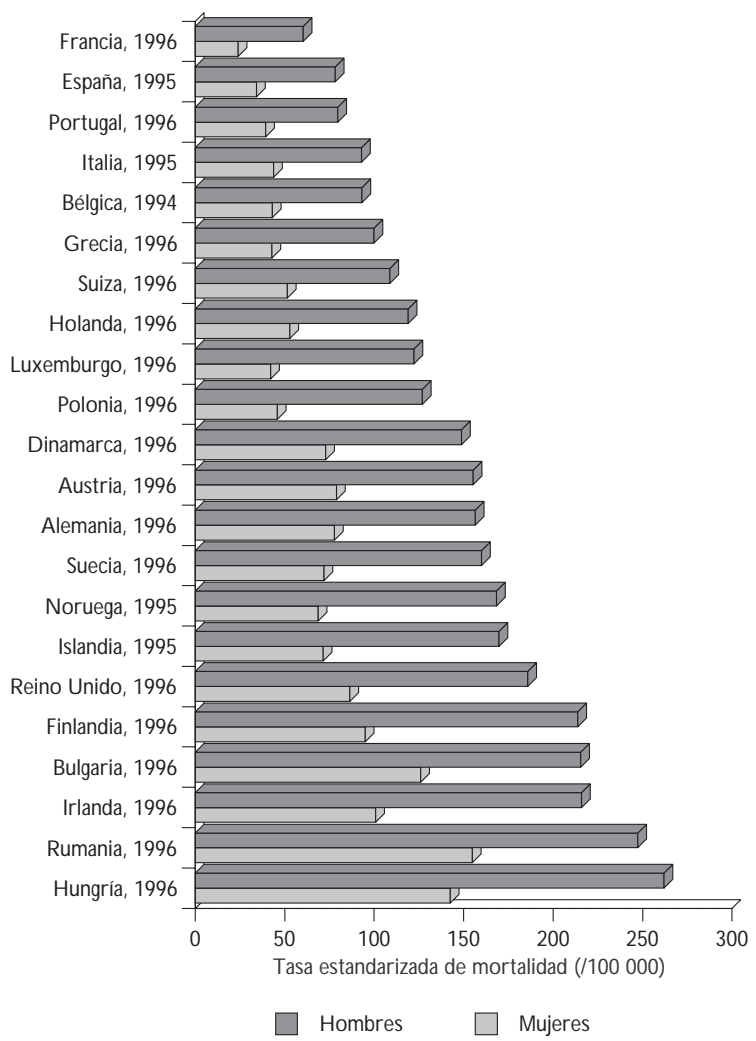

Fuente: referencia 7

Figura 1. Mortalidad por enfermedad isquémica del CORAZÓN EN 22 PAÍSES EUROPEOS 


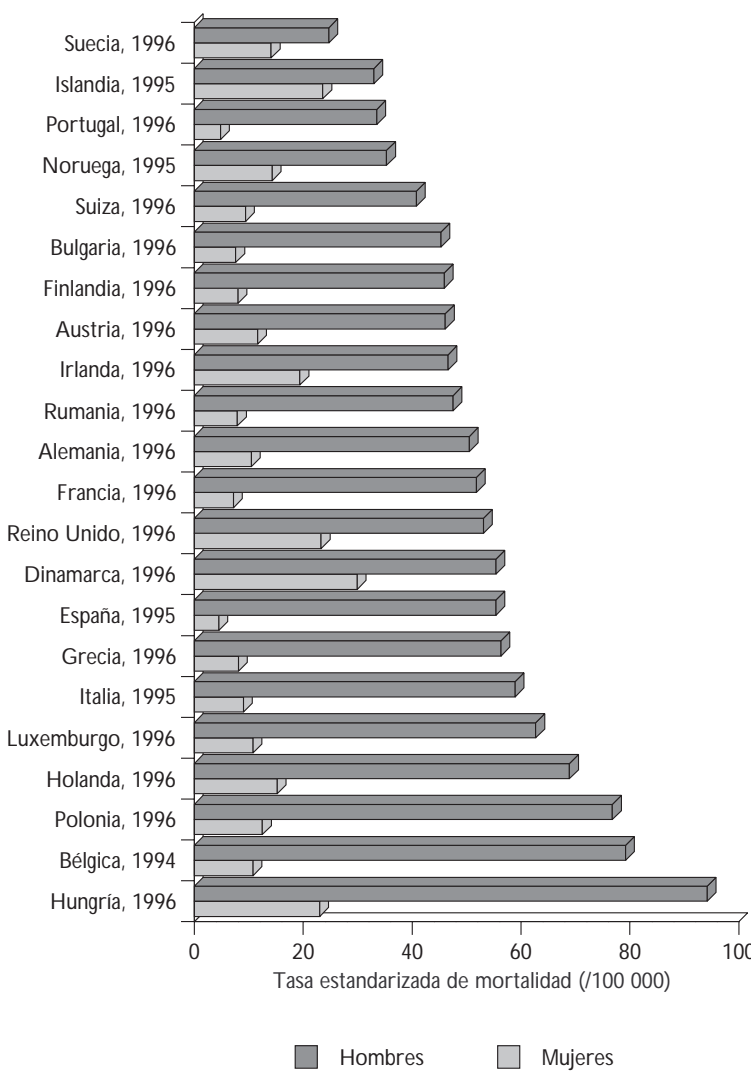

Fuente: referencia 7

Figura 2. MORTALIDAd POR CÁNCER DE TRÁQUEA, BRONQUIOS Y PULMÓN EN 22 PAÍSES EUROPEOS

superiores en los países del centro, este y norte de Europa (Hungría, Rumania, Irlanda, Bulgaria y Finlandia), en comparación con las tasas en los países del sur de Europa (Francia, España, Portugal o Italia). En relación con el cáncer de pulmón (figura 2), existe un rango de variación entre 4 y 5 en los países con tasas más elevadas (Hungría, Bélgica y Polonia en los hombres, y Dinamarca, Islandia y Reino Unido en las mujeres); los países con tasas más bajas (Suecia, Islandia y Portugal en los hombres, y España, Portugal y Francia en las mujeres).

Este patrón global de mortalidad encubre un importante efecto cohorte en los diferentes países, y deben considerarse las tendencias en las últimas décadas. Así, puede apreciarse una tendencia favorable con descenso de la mortalidad por enfermedad isquémica del corazón en la mayoría de países del norte de Europa. La mortalidad por cáncer de pulmón en hombres muestra una cierta estabilización en la mayoría de países del centro y sur de Europa, un claro descen- so en los países nórdicos y más occidentales, y un aumento en los países del este, que presentan las mayores tasas en hombres de mediana edad. ${ }^{9}$ En las mujeres, sin embargo, se observa una tendencia desfavorable al aumento de la mortalidad en la mayoría de países europeos. ${ }^{10}$ Estas tendencias no hacen sino reflejar la necesidad urgente de poner en marcha medidas para el control de la mortalidad relacionada con el tabaco -la prevención del tabaquismo entre los jóvenes y la promoción del abandono, sobre todo en mujeres y en los países del sur y este de Europa -, como veremos en el apartado siguiente.

\section{Prevalencia de tabaquismo}

La comparación de la prevalencia de tabaquismo entre diferentes países es problemática, puesto que suele provenir de encuestas con diferente metodología en las que puede variar la representatividad, la definición de fumador, los métodos de recolección de datos, etcétera. Recientemente se han publicado los resultados de las encuestas sobre factores de riesgo cardiovascular de las poblaciones participantes en el proyecto WHO MONICA. ${ }^{11}$ Estas encuestas se realizaron siguiendo la misma metodología estandarizada en los diferentes países participantes, por lo que su comparabilidad está libre -en buena parte- de los potenciales sesgos antes mencionados.

La figura 3 muestra la prevalencia de fumadores y fumadoras diarios, estandarizada por edad, para 12 de las poblaciones europeas participantes en el estudio WHO MONICA, a mediados de los años 1980 y 1990. La figura, además, incluye la tendencia, expresada como cambio promedio de la prevalencia en la década de estudio. En el grupo de países considerados, las prevalencias más elevadas a mediados de los años 80 se registraban en Varsovia-Polonia (59\%), GlasgowReino Unido (52\%), Charleroi-Bélgica (51\%), y Cataluña-España (48\%). La prevalencia de tabaquismo ha disminuido en todas las poblaciones mostradas en los hombres, con disminuciones entre 12.4 puntos en Brianza-Italia y 1.9 en Glostrup-Dinamarca. A mediados de los años 90 sólo cinco poblaciones tuvieron una prevalencia en hombres inferior a 30\%. Esta disminución es debida a la elevada proporción de nunca fumadores en los grupos de edad más jóvenes más que al abandono del hábito en las edades intermedias. ${ }^{11}$ En las mujeres, las prevalencias más elevadas se registraban en el Reino Unido (50\%) y Dinamarca (44\%), y en siete poblaciones la prevalencia era inferior a $25 \%$ a mediados de los años 80 . La prevalencia se ha reducido en aquellas poblaciones en las que ya era elevada al inicio del periodo estudiado, mientras que ha aumen- 


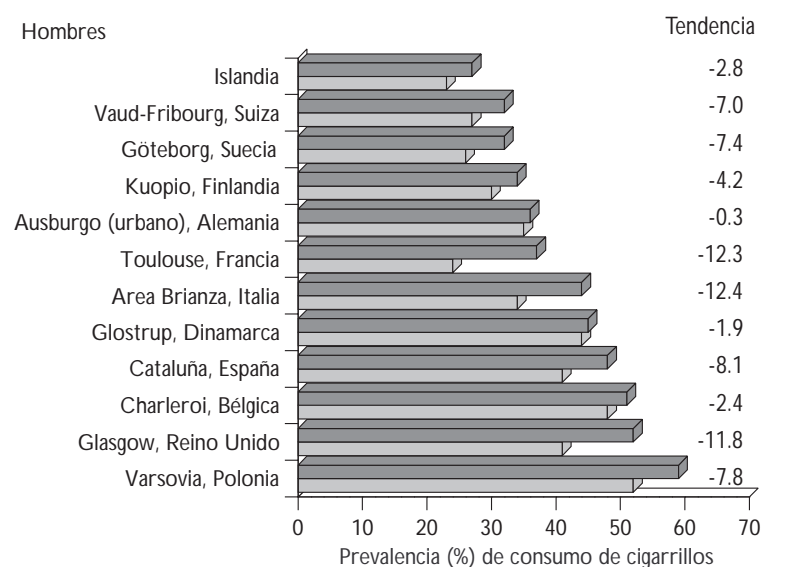

Años 1980* $\square$ Años 1990*

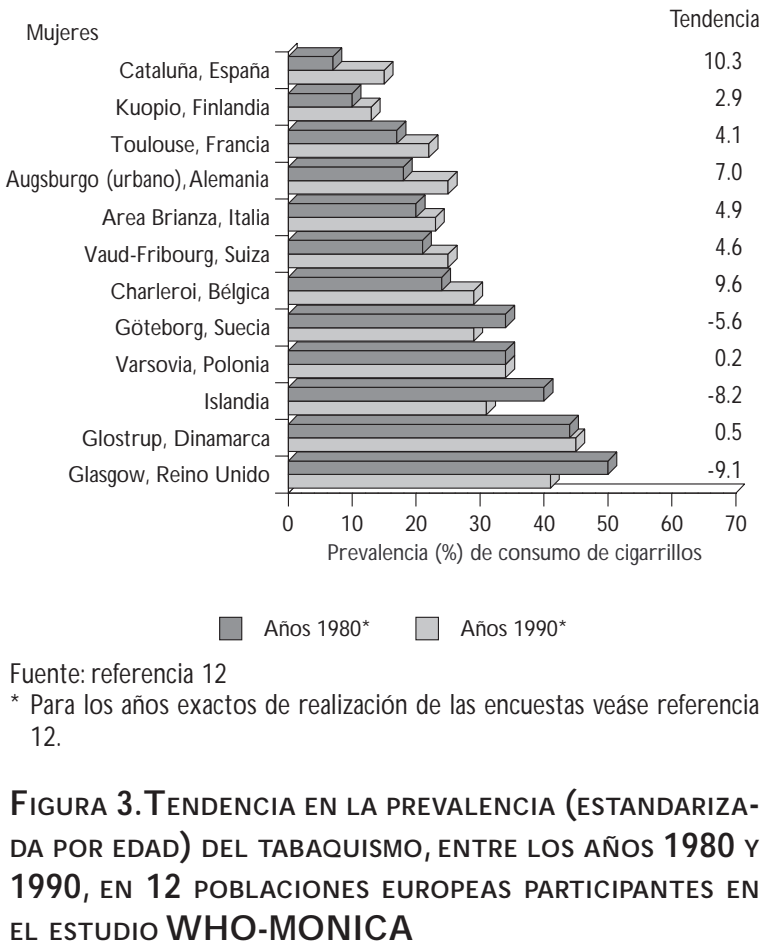

tado en las poblaciones que presentaban una prevalencia inicial más baja.

Según estimaciones derivadas del Proyecto "Tabaco o Salud", la prevalencia de consumo de cigarrillos en la región europea de la OMS era $43.5 \%$ en hombres y $23.4 \%$ en mujeres, lo que representa aproximadamente unos 229 millones de fumadores (144.3 millones de hombres y 84.7 millones de mujeres). Esta estimación se ha basado en el metanálisis de 40 estu- dios que representan aproximadamente a $90 \%$ de la población de la región europea. ${ }^{12}$

Otro estudio con información sobre tabaquismo en 12 países europeos, referida a inicios de los años 90, muestra que existen diferencias en las prevalencias según el género y el nivel educativo. ${ }^{13}$ Se observa una mayor prevalencia de tabaquismo en las mujeres con menor nivel de estudios en el Reino Unido, Noruega y Suecia, mientras que en los países del sur de Europa la proporción de fumadoras es mayor entre las de mayor nivel educativo. Se observa un patrón similar nortesur, aunque menos acentuado, en la prevalencia según el nivel educativo entre los hombres. ${ }^{13}$ Además, la manera de fumar de hombres y mujeres es diferente; las mujeres fuman menos cigarrillos al día que los hombres, prefieren los cigarrillos con filtro, el tabaco rubio y los cigarrillos bajos en nicotina, y no suelen fumar cigarrillos de liar, ni puros ni pipas. ${ }^{14}$

De acuerdo con esta información sobre la prevalencia y sus tendencias podemos clasificar las poblaciones estudiadas en diferentes fases del modelo descriptivo de la epidemia de tabaquismo propuesto por López y colaboradores. ${ }^{4}$ Las poblaciones del sur de Europa y algunas poblaciones centro y norte-europeas presentan una disminución de la prevalencia en los hombres pero no en las mujeres, incluso con aumento de la prevalencia en estas últimas, típica de la fase III. Las poblaciones del Reino Unido y otros países como Islandia o Suecia, presentan características de la fase IV, con una disminución importante de la prevalencia tanto en hombres como en mujeres. ${ }^{11}$ Estas diferencias en la prevalencia en los países no hacen sino expresar el diferente patrón de inicio y de abandono del hábito tabáquico en cada uno, como varios trabajos más detallados han mostrado en algunas poblaciones europeas. ${ }^{15-19}$

\section{Tabaquismo pasivo}

Es bien conocido que la exposición al humo del tabaco ambiental, o tabaquismo pasivo, (también conocido como 'aire contaminado por humo de tabaco'), se ha asociado tanto con efectos agudos como crónicos sobre la salud..$^{20}$ La exposición al humo ambiental del tabaco puede ocurrir tanto en los lugares de residencia (en los propios domicilios de los no fumadores) como en los lugares de trabajo, además de otros lugares públicos o privados (zonas recreativas, de ocio, etcétera). En los Estados Unidos de América, 37\% de la población adulta no fumadora declara compartir espacios con humo ya sea en el lugar de trabajo o en el propio domicilio. ${ }^{21}$ A pesar de ser una exposición emer- 
gente, no existe en la actualidad información estandarizada y comparable de base poblacional sobre exposición pasiva al humo del tabaco en los países europeos. Como aproximación a la prevalencia de exposición al humo ambiental del tabaco podemos utilizar la información procedente del grupo control de un estudio de casos y controles multicéntrico realizado en 12 zonas de siete países europeos, coordinado por la Agencia Internacional de Investigación sobre el Cáncer (IARC). ${ }^{22}$ Estos grupos control fueron de base poblacional en siete centros y de base hospitalaria (acompañantes de pacientes o pacientes visitados en los mismos hospitales que los casos) en el resto de centros. La exposición pasiva al tabaco, laboral o doméstica, fue $78 \%$ en el conjunto de los controles.

Más recientemente, la Encuesta Europea sobre Salud Respiratoria para investigar la prevalencia de asma en adultos jóvenes (20-44 años) también ha aportado información relevante sobre la exposición al humo ambiental del tabaco en diferentes países europeos. ${ }^{23}$ Como mostraba el estudio anterior, existe una gran variación entre las diferentes ciudades europeas donde se realizó el estudio, con una prevalencia de exposición (laboral o doméstica) elevada en Galdakao (75.9\%) o Huelva (67.3\%) en España, Pavia (61.8\%) en Italia, o Dublín (63.2\%) en Irlanda, y considerablemente menor en ciudades suecas como Uppsala (21.5\%) o Umea (18.9\%). La mayor parte de ciudades donde se realizó el estudio mostraron prevalencia de exposición pasiva entre 30 y $50 \%$.

Las autoridades sanitarias europeas, conscientes del gran problema que la exposición pasiva al tabaco representa, han emprendido actividades para desarrollar políticas conjuntas de carácter legislativo y educativo de control del tabaquismo pasivo, y de promoción de ambientes libres de humo. ${ }^{24}$ En el mismo sentido, la OMS dedica el primer "Día Mundial sin Tabaco" del milenio al tabaquismo pasivo, bajo el lema 'Humo de segunda mano: limpiemos el aire'. ${ }^{25}$

\section{Producción de tabaco}

La producción anual de tabaco en toneladas no manufacturado en cada país, junto con las toneladas de tabaco importadas y dedicadas a la exportación durante 1994 en Europa, se presenta en el cuadro I, elaborado a partir del Informe "Tabaco o Salud", de la OMS. ${ }^{8}$ En términos absolutos, y teniendo en cuenta las toneladas anuales de tabaco producido o importado para su elaboración, la mayor actividad se concentra en el sur de Europa (Grecia, Italia y España), junto con el Reino Unido y Alemania. Este país es el quinto productor mundial de cigarrillos, tras China, Estados Unidos de

\section{Cuadro I \\ Producción anual (toneladas) de tabaco no manufacturado en 21 países eUropeos, 1994}

Producción Importación Exportación

\begin{tabular}{lccc}
$\begin{array}{l}\text { Unión Europea } \\
\text { Alemania }\end{array}$ & 9000 & 196000 & 26000 \\
\hline Austria & 350 & 12500 & - \\
\hline Bélgica & 1300 & 35000 & 6000 \\
\hline Dinamarca & - & 16400 & - \\
\hline España & 44000 & 61000 & 16700 \\
\hline Finlandia & - & 6000 & - \\
\hline Francia & 26900 & 44000 & 6500 \\
\hline Grecia & 156000 & 15000 & 110000 \\
\hline Holanda & - & 94370 & - \\
\hline Irlanda & - & 5500 & - \\
\hline Italia & 134000 & 26000 & 110000 \\
\hline Luxemburgo & No disponible & & \\
\hline Portugal & 5446 & 8000 & 2000 \\
\hline Reino Unido & - & 134000 & - \\
\hline Suecia & - & 10000 & - \\
& & & \\
O tros países de la Europa del 0 este & & & \\
N oruega & - & 4600 & - \\
\hline Suiza & 1400 & 30750 & 7700
\end{tabular}

Selección de países de Europa del Este

\begin{tabular}{lccc} 
Bulgaria & 30000 & - & - \\
\hline Hungría & 13800 & 8000 & 1400 \\
\hline Polonia & 43000 & 25000 & - \\
\hline Rumania & 11400 & 9500 & -
\end{tabular}

Fuente: referencia 9

América y Japón. ${ }^{26}$ La producción de tabaco de Grecia e Italia se destina principalmente a la exportación, dirigida en buena parte a países del centro y este de Europa, así como del norte de Africa. Además de importar una gran cantidad de tabaco no manufacturado, Bélgica es un importante importador de cigarrillos manufacturados de los Estados Unidos de América, con destino a los mercados legales e ilegales de los países del sur y este de Europa. ${ }^{8}$

El peso de Europa en el mercado mundial del tabaco es importante, como lo demuestra que 10 de sus países (Alemania, Rusia, Reino Unido, Polonia, Países Bajos, España, Grecia, Italia, Bulgaria y Francia) se encuentran entre los 25 principales productores de tabaco del mundo. En estos países se maneja 13\% de los cerca de siete millones de toneladas de tabaco usado 
aparentemente por los manufactureros en el ámbito mundial (como balance de la producción, importación, exportación y reservas). ${ }^{26}$

\section{Consumo de cigarrillos}

El cuadro II, elaborado también a partir de la información recopilada por la OMS, ${ }^{8,26}$ muestra la evolución del consumo de cigarrillos anual per capita en la población $\geq$ de 15 años para los 22 países europeos seleccionados desde los años 70. Como puede observarse, el patrón de consumo de tabaco refleja el patrón establecido a partir de la prevalencia del tabaquismo. El

\section{Cuadro II \\ Consumo anual per capita en adultos (POBLACIÓN $\geq 15$ AÑOS), DE CIGARRILLOS MANUfACTURADOS, EN 22 PAÍSES EUROPEOS, 1970-1992}

$\begin{array}{cccc} & & & \text { \% de cambio } \\ & \text { Año } & 1990-92 \text { vs } \\ 1970-72 & 1980-82 & 1990-92 & 1970-72\end{array}$

\begin{tabular}{lcccc}
$\begin{array}{l}\text { Unión Europea } \\
\text { Alemania }\end{array}$ & 2430 & 2420 & 2360 & -2.88 \\
\hline Austria & 2390 & 2620 & 2210 & -7.53 \\
\hline Bélgica & 3090 & 2880 & 2310 & -25.24 \\
\hline Dinamarca & 2050 & 2020 & 1919 & -6.39 \\
\hline España & 2190 & 2440 & 2670 & +21.92 \\
\hline Finlandia & 2000 & 1800 & 1740 & -13.0 \\
\hline Francia & 1860 & 2080 & 2120 & +13.98 \\
\hline Grecia & 2640 & 3440 & 3590 & +35.98 \\
\hline Holanda & 3150 & 3290 & 2820 & -10.48 \\
\hline Irlanda & 3050 & 3030 & 2420 & -20.66 \\
\hline Italia & 1800 & 2310 & 1920 & +6.67 \\
\hline Luxemburgo & 3090 & 2580 & 2080 & -32.68 \\
\hline Portugal & 1440 & 1800 & 2010 & +39.58 \\
\hline Reino Unido & 3250 & 2740 & 2210 & -32.0 \\
\hline Suecia & 1700 & 1840 & 1550 & -8.82
\end{tabular}

0 tros países de la Europa del 0 este

\begin{tabular}{lllll} 
Islandia & 2940 & 3.230 & 2.860 & -2.72 \\
\hline N oruega & 2030 & 1.950 & 1.830 & -9.85 \\
\hline Suiza & 3700 & 3.060 & 2.910 & -21.35
\end{tabular}

Selección de países de Europa del Este

\begin{tabular}{lllll} 
Bulgaria & 1770 & 1880 & 2240 & +26.55 \\
\hline Hungría & 2940 & 3320 & 3260 & +10.88 \\
\hline Polonia & 3010 & 3400 & 3620 & +20.26 \\
\hline Rumania & 1740 & 2130 & 1550 & -10.91
\end{tabular}

Fuente: referencia 9 consumo de cigarrillos per capita ha disminuido considerablemente en la mayoría de países de la Unión Europea, excepto en Francia, Grecia, Portugal y España, países en los que, al contrario, el consumo ha aumentado entre 1970 y 1990. El consumo ha aumentado también considerablemente en los países del este. Son estos países, y los ya mencionados del sur de Europa, los que presentan un consumo más elevado de cigarrillos per capita. La evolución más detallada del consumo para algunos países seleccionados, representativos de las tendencias observadas, puede apreciarse en la figura 4.

Es importante hacer notar que la información presentada se refiere al consumo anual de cigarrillos per capita de la población adulta ( $\geq 15$ años). Ello conlleva una importante limitación, como es la falta de poder para discriminar el consumo de cigarrillos por sexos. En publicaciones precedentes, el análisis del consumo se ha basado bien en el número de cigarrillos o los gramos de tabaco consumidos por día, 5,6 por lo que la comparabilidad de la información es limitada.

El uso de información referente a producción (producción más importaciones, menos exportaciones) de cigarrillos, además, puede infraestimar el consumo en algunos países (como Holanda, Bélgica o Dinamarca) debido al elevado consumo de cigarrillos liados (hand rolled cigarettes o roll-your-own cigarettes), ${ }^{27}$ al consumo de tabaco comprado en otros países (como es el caso de Bélgica y Luxemburgo), o al contrabando de tabaco entre países (como en Italia y España), al que nos referiremos más adelante. ${ }^{28,29}$

\section{Precio, impuestos y contrabando de tabaco}

Uno de los mayores determinantes del consumo de cigarrillos es su precio, por lo que los impuestos que gravan el precio del tabaco son una de las maneras más efectivas de reducir el consumo. ${ }^{30-32}$ En el cuadro III se muestran los precios (en dólares USA) al consumidor y los impuestos totales asociados de una cajetilla de 20 cigarrillos, de la marca más popular, en los países de la Unión Europea. ${ }^{33}$ Los países del sur de Europa (España, Portugal, Italia y Grecia) presentan los precios más bajos, mientras que los más elevados corresponden al Reino Unido e Irlanda. Los impuestos representan entre 70 y $86 \%$ del precio final del tabaco. El precio real de los cigarrillos, ajustado por la inflación, ha aumentado en la mayor parte de países europeos durante la última década, especialmente para los elaborados en cada país, mientras que para los importados los precios se han mantenido estables. ${ }^{12} \mathrm{El}$ precio de los cigarrillos es diferente en los países europeos, aun teniendo en cuenta el costo de la vida. ${ }^{31}$ 

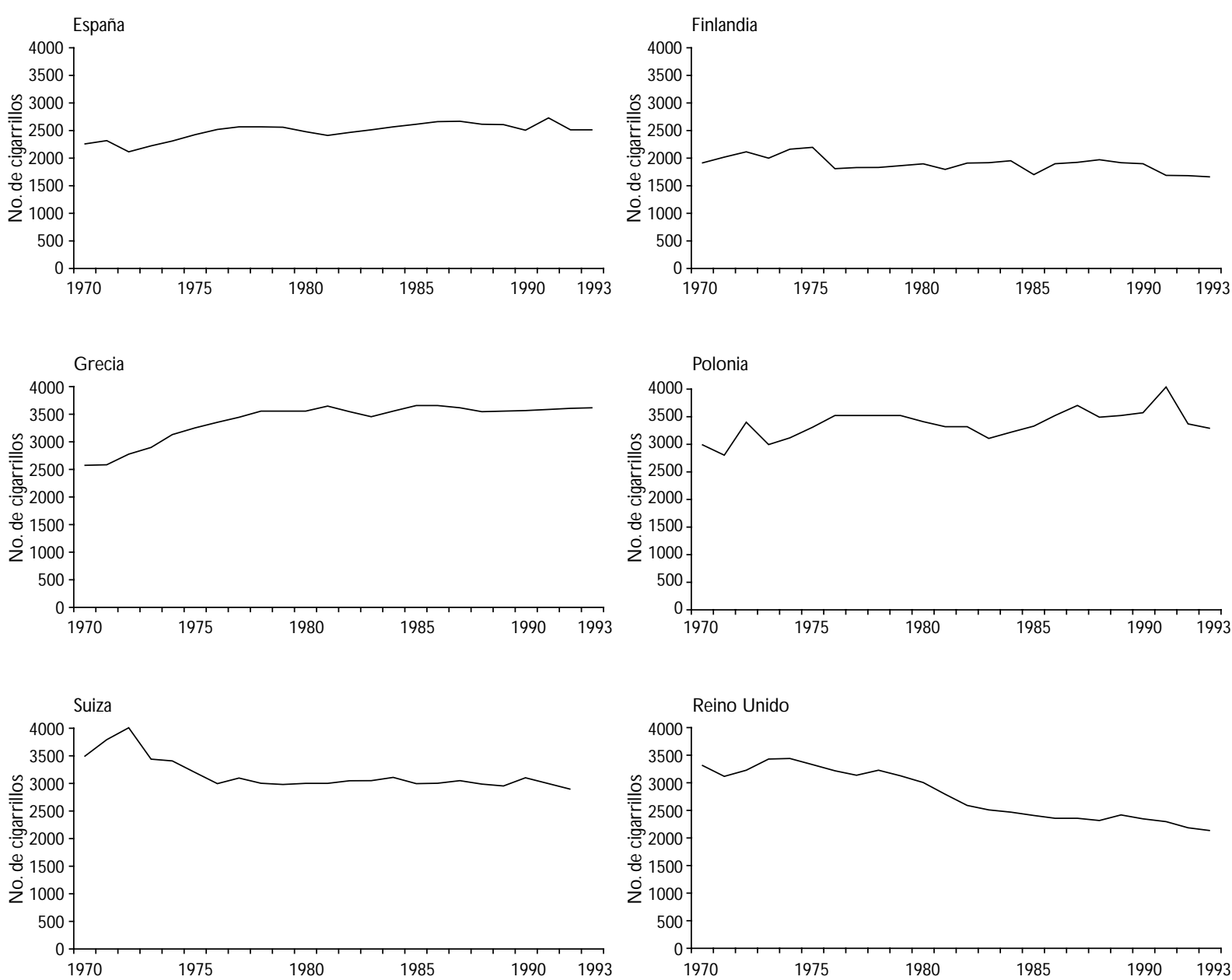

Fuente: Elaborado a partir de la referencia 9

Fuente: referencia 9

Figura 4. Consumo anual per capita en adultos (población $\geq 15$ años), de cigarrillos manufacturados, en España, Finlandia, Grecia, Polonia, Reino Unido y Suiza, 1970-1990

La ley de la oferta y la demanda indica que cuanto más caro es un producto menos personas lo comprarán. En un estudio que incluía información sobre 27 países europeos la elasticidad del precio de demanda (es decir, el porcentaje de cambio en el consumo de cigarrillos por $1 \%$ de cambio en el precio) fue de 0.4 , es decir, que el aumento del precio del tabaco supone una reducción considerable de su consumo. ${ }^{35}$ Además, se ha observado que la elasticidad del precio del tabaco es mayor en los grupos sociales más desfavorecidos. ${ }^{36}$ Estas investigaciones ponen de manifiesto que el aumento de los precios del tabaco anima a ciertas personas a dejar de fumar y que puede pre- venir, por motivos económicos, el inicio del hábito en jóvenes o la recaída de ex fumadores. Además, una pequeña proporción de los impuestos del tabaco sería suficiente para financiar las actividades de control del tabaco en Europa, e incluso las actividades culturales y deportivas que en la actualidad todavía patrocina la industria del tabaco. ${ }^{37}$

A pesar de ello, la industria tabaquera insiste en su lucha contra los impuestos sobre el tabaco, y uno de sus principales argumentos radica en el contrabando de éste. Según la industria, el contrabando se produce a causa de las diferencias de precio (debidas sobre todo a la diferente carga fiscal sobre el tabaco) 


\section{Cuadro III \\ Precios e impuestos totales (en dólares USA) DE UN PAQUETE DE 20 CIGARRILLOS DE LA MARCA más popular en los países de la Unión Europea}

Precio (US \$) Impuestos (US \$) Razón precio/ impuestos

\begin{tabular}{llll} 
Reino Unido & 5.32 & 4.58 & 0.86 \\
\hline Irlanda & 4.54 & 3.63 & 0.80 \\
\hline Suecia & 4.14 & 2.90 & 0.70 \\
\hline Dinamarca & 3.91 & 3.20 & 0.82 \\
\hline Finlandia & 3.59 & 2.73 & 0.76 \\
\hline Francia & 2.87 & 2.18 & 0.76 \\
\hline Alemania & 2.61 & 1.85 & 0.71 \\
\hline Bélgica & 2.54 & 1.90 & 0.75 \\
\hline Holanda & 2.36 & 1.70 & 0.72 \\
\hline Austria & 2.25 & 1.67 & 0.74 \\
\hline Grecia & 1.89 & 1.38 & 0.73 \\
\hline Italia & 1.85 & 1.39 & 0.75 \\
\hline Portugal & 1.69 & 1.35 & 0.80 \\
\hline España & 1.63 & 1.16 & 0.71
\end{tabular}

Fuente: referencia 32

entre países donde es "más barato" y donde es "más caro" ${ }^{29}$ Pero el contrabando es más prevalente en los países donde es "más barato", como es el caso de España. Además, en los países donde se han reducido los impuestos supuestamente para reducir el contrabando, como en Suecia o Canadá, el consumo ha aumentado de forma alarmante. A pesar de la insistencia de la industria, ha quedado demostrado que el contrabando no se reduce a una actividad "turística" o de aficionados entre países vecinos, sino que existe un contrabando a gran escala (contrabando de "containers") que parece representar un sector más del mercado de las grandes compañías tabaqueras. Por ello, la Comisión Europea ha planteado una demanda legal contra las compañías tabaqueras por el tráfico ilegal.

El problema del contrabando se puede controlar mediante medidas específicas entre los gobiernos de los países implicados, como ha quedado demostrado recientemente en el caso de España con el tabaco de contrabando procedente de Andorra, que también era introducido en otros países de la Unión Europea. ${ }^{38}$

\section{Conclusiones}

Aproximadamente 30 de cada 100 europeos adultos fuman cigarrillos a diario. En los países de la Unión Europea, se estima que uno de cada 10 adultos fuma- dores morirá a causa de los efectos del tabaco, razón que se eleva a uno de cada cinco en los países del este de Europa. La prevalencia de fumadores desciende entre los hombres adultos en algunos países de la Unión Europea, mientras que aumenta en las mujeres, sobre todo jóvenes, del sur y del este de Europa. Por todo lo expuesto, el único modo de erradicar la epidemia de tabaquismo -y sus consecuencias en los fumadores y en los no fumadores- es insistir en las medidas para controlar el consumo de tabaco y prevenirlo: acciones educativas, clínicas, reglamentarias, económicas y globales. ${ }^{1,39}$ Entre ellas, cabe destacar la prohibición del consumo de tabaco en lugares públicos, la prohibición real de la publicidad directa e indirecta del tabaco, la reducción del cultivo del tabaco, o el incremento de las políticas de precios. Estas acciones se deben diseñar, coordinar e implantar en y desde los diferentes sectores involucrados en la lucha contra el tabaco -educación, salud pública, economía, industria, justicia- según sea oportuno y con la participación de redes civiles (organizaciones no gubernamentales, de afectados, grupos de presión, etcétera). $Y$ deben ser animadas desde los diferentes niveles administrativos (local, regional, nacional) con la decisiva participación de los organismos y organizaciones supranacionales. ${ }^{1,25}$

\section{Referencias}

1. Clemente P, Fleitmann S, Hirsch A. Lisbon declaration 2000 on smoking prevention in Europe. Lisboa: European N etwork for Smoking Prevention; 2000. Disponible en: URL: http://www.ensp.org/uk/declaration.

2. Peto R, López AD, Boreham J,Thun M, Heath C, D oll R. Mortality from smoking worldwide. Br Med Bull 1996 Jan;52(1):12-21.

3. World Health 0 rganization. Smoking and premature adult mortality. W orld health Statistics A nnual. Ginebra:W orld Health 0 rganization, 1990. 4. López AD, Collishaw NE, Piha T.A descriptive model of the cigarette epidemic in developed countries. Tobacco Control 1994;3:242-247.

5. Hill C. Trends in tobacco use in Europe. J Natl Cancer Inst Monogr 1992;12:21-24.

6. Franceschi $\mathrm{S}, \mathrm{N}$ aett $\mathrm{C}$. Trends in smoking in Europe. Eur J $\mathrm{C}$ ancer Prev $1995 ; 4: 271-284$.

7. Mortality data. 0 nline version of the World Health Statistics Annual. W HO Statistical Information System. Disponible en: URL: http:// www.who.int/whosis/.

8.Tobacco or Health Programme.W orld Health 0 rganization. Tobacco or Health:A G lobal Status Report. Country Profiles by Region, 1997. 0 ffice on Smoking and Health of the CDC's $\mathrm{N}$ ational $\mathrm{C}$ enter for Chronic Disease Prevention and Health Promotion. Disponible en: URL: http:// www.cdc.gov/tobacco/ who/whofirst.htm.

9. LaVecchia C, Levi F, Lucchini F, N egri E.Trends in mortality from major diseases in Europe, 1980-1993. Eur J Epidemiol 1998;1:1-8.

10. La Vecchia C, Boyle P, Franceschi S, Levi F, Maisonneuve P, N egri E et al. Smoking and cancer with emphasis on Europe. Eur J C ancer 1991;27: 94-104. 
11. Molarius A, Parsons RW, D obson AJ, Evans A, Fortmann SP, Jamrozik K et al. Trends in cigarette smoking in 36 populations from the early $1980 \mathrm{~s}$ to the Mid-1990: Findings from the W HO MO N ICA Project. Am J Public Health 2001;91:206-212.

12. Corrao MA, G uindon GE, Cokkinides V, Sharma N. Building the evidence base for global tobacco control. Bull W HO 2000;78:884-890.

13. C avelaars AEJM, Kunst AE, G eurts JJM, C rialesi R, Grötvedt L, Helmert $U$ et al. Educational differences in smoking: International comparison. BMJ 2000;320:1102-1107

14. Joossens L. La igualdad se va con el humo. Las mujeres y el tabaco en la Unión Europea. Bruselas: European N etwork for Smoking Prevention, 1999.

15. La Vecchia C, D ecarli A, Pagano R. Prevalence of cigarette smoking among subsequent cohorts of Italian males and females. Prev Med 1986;15:606-613.

16. La Vecchia C, Levi F, Decarli A, W ielisbach V, N egri E, Gutzwiller F. Trends in smoking and lung cancer mortality in Switzerland. Prev Med 1988;17:712-714.

17. Brenner H.A birth cohort analysis of the smoking epidemic in W est Germany.J Epidemiol Community Health 1993;47:54-58.

18. Laaksonen M, U utela A,Vartiainen E, Jousilahti P, Helakorpi S, Puska P. Development of smoking by birth cohort in the adult population in eastern Finland 1972-97. Tobacco Control 1999;8:161-168.

19. Borrás JM, Fernández E, Schiaffino A, Borrell C, LaVecchia C. Pattern of smoking initiation in Catalonia (Spain) from 1948 to 1992. Am J Public Health 2000;90:1459-1462.

20. US Department of Health and Human Services. The health consequences of involuntary smoking:A report of the surgeon general. Washington DC: US Department of Health and Human Services, $O$ ffice of Smoking and Health, 1986. Publication PHS 87-8398.

21. Pirkle JL, Flegal KM, Bernert JT, Brody DJ, Etzel RA, Maurer KR. Exposure of the US population to environmental tobacco smoke. The Third $\mathrm{N}$ ational Health and N utrition Examination Survey, 1988 to 1991. JAMA 1996,275:1233-1240.

22. Boffetta P,A gudo A,A hrens W, Benhamou E, Benhamou S, D arby SC et al. European multicenter case-control study of lung cancer in non-smokers. Lyon: International A gency for Research on Cancer; 1998. IARC Technical Report $N 033$.

23. Janson C, Chinn S, Jarris D, Zock JP,Torén K, Burney P. For the European Community Respiratory Health Survey. Lancet 2001;358:2103-2109.
24. World Health 0 rganization. Policies to reduce exposure to environmental tobacco smoke. Report on a W HO working group meeting. C Openhagen:W orld Health 0 rganization, 2000.

25. W orld Health 0 rganization. Tobacco Free Inititative. URL: http:// tobacco.who.int/en/ advocacy/wntd2001a.html.

26. Collishaw NE, López AD, ed. Tobacco alert. Ginebra: World Health 0 rganization, 1996. Disponible en: URL: www.who.ch/programmes/psa/ toh.htm.

27. European Bureau for Action on Smoking Prevention. Tobacco and Health in the European Union. An overview. Bruselas: BASP, 1994.

28. $N$ icolaides-Bouman $A, W$ ald N , Forey $B$, Lee P. International smoking statistics. A collection of historical data from 22 economically developed countries. 0 xford: 0 xford University Press, 1993.

29. Joosens L, Raw M. Smuggling and cross border shopping of tobacco in Europe. BMJ 1995;310:1393-1397.

30. G odfrey C , Maynard A. Economic aspects of to bacco use and taxation policy. BMJ 1988;297:339-343.

31. Jha P, C haloupka FJ. C urbing the epidemic: G overnments and the economics of tobacco control.W ashington DC:The W orld Bank, 1999

32. Townsend J. Price and consumption of tobacco. Br Med Bull 1996;52: 132-142.

33. N on-smokers' Rights Association. Smoking and Health Action Foundation. G lobal cigarette taxes and prices. D isponible en:URL:http://www.nsraadfn.ca/ english/staxratesus.html.

34. Montes A, Villalbi JR.The price of cigarettes in the European Union.Tob Control 2001;10(2):135-136.

35. Townsend J. Price, tax, and smoking in Europe. Copenhagen: World Health 0 rganization, 1988.

36. Townsend J, Roderick P, Cooper J. Cigarette smoking by so cioeconomic group, sex, and age: Effects of price, income, and healthy publicity. BMJ 1994;309:923-927.

37. Jamison DT, C reese A, Prentice T, ed.The W orld Health Report 1999 Making a difference. G inebra:W orld Health 0 rganization: 1999;65-79. 38. Joossens L, Raw M. How can cigarette smuggling be reduced? BMJ 2000;321:947-950.

39. Centers for Disease Control and Prevention. Reducing to bacco use:A report of the Surgeon General-executive summary. MMW R Morb Mortal W kly Rep 2000;49(RR-16):1-27. 